\title{
Synergistic Activity of Capsaicin and Colistin Against Colistin-Resistant Acinetobacter baumannii: In Vitro/Vivo Efficacy and Mode of Action
}

\author{
Tingting Guo ${ }^{1,2,3}$, Mengying $\mathrm{Li}^{1,4}$, Xiaoli Sun ${ }^{1}$, Yuhang Wang ${ }^{1}$, Liying Yang ${ }^{1}$, Hongmei Jiao ${ }^{1}$ \\ and Guocai $\mathrm{Li}^{*},{ }^{1,2,3}$ \\ ${ }^{1}$ Department of Microbiology, School of Medicine, Yangzhou University, Yangzhou, China, ${ }^{2}$ Jiangsu Key Laboratory of Zoonosis/ \\ Jiangsu Co-Innovation Center for Prevention and Control of Important Animal Infectious Diseases and Zoonoses, Yangzhou \\ University, Yangzhou, China, ${ }^{3}$ Jiangsu Key Laboratory of Integrated Traditional Chinese and Western Medicine for Prevention and \\ Treatment of Senile Diseases, Yangzhou, China, ${ }^{4}$ Department of Pharmacy, Suzhou Hospital of Integrated Traditional Chinese \\ and Western Medicine, Suzhou, China
}

Acinetobacter baumannii is an opportunistic pathogen predominantly associated with nosocomial infections. With emerging resistance against polymyxins, synergistic combinations of drugs are being investigated as a new therapeutic approach.

OPEN ACCESS

Edited by:

Wangxue Chen,

National Research Council Canada (NRC-CNRC), Canada

Reviewed by:

Frank Schweizer, University of Manitoba, Canada

Suzana Katarina Straus,

University of British Columbia, Canada

*Correspondence:

Guocai Li

gcli@yzu.edu.cn

Specialty section:

This article was submitted to Pharmacology of Infectious Diseases,

a section of the journal

Frontiers in Pharmacology

Received: 20 July 2021 Accepted: 03 September 2021 Published: 17 September 2021

Citation:

Guo T, Li M, Sun X, Wang Y, Yang L, Jiao $H$ and Li G (2021) Synergistic Activity of Capsaicin and Colistin Against Colistin-Resistant Acinetobacter baumannii: In Vitro/Vivo

Efficacy and Mode of Action.

Front. Pharmacol. 12:744494.

doi: 10.3389/fphar.2021.744494
Capsaicin is a common constituent of the human diet and is widely used in traditional alternative medicines. The present study evaluated the antibacterial activities of capsaicin in combination with colistin against three unrelated colistin-resistant Acinetobacter baumannii strains in vitro and in vivo, and then further studied their synergistic mechanisms. Using the checkerboard technique and time-kill assays, capsaicin and colistin showed a synergistic effect on colistin-resistant $A$. baumannii. A mouse bacteremia model confirmed the in vivo effects of capsaicin and colistin. Mechanistic studies shown that capsaicin can inhibit the biofilm formation of both colistin-resistant and non-resistant $A$. baumannii. In addition, capsaicin decreased the production of intracellular ATP and disrupted the outer membrane of $A$. baumannii. In summary, the synergy between these drugs may enable a lower concentration of colistin to be used to treat A. baumannii infection, thereby reducing the dose-dependent side effects. Hence, capsaicin-colistin combination therapy may offer a new treatment option for the control of $A$. baumannii infection.

Keywords: Acinetobacter baumannii, capsaicin, colistin, combination, mechanisms

\section{INTRODUCTION}

The emergence of antibiotic resistance poses a serious threat to global health. The overuse and abuse of existing antibiotics have resulted in concerning levels of bacterial resistance and loss of the therapeutic effectiveness of many drugs (Yoshida et al., 2017). Acinetobacter baumannii is a non-fermenting Gram-negative opportunistic pathogen that is widely distributed in clinical settings. This bacterium causes a variety of infections in patients with low immunity, including bacteremia and ventilator-associated pneumonia (Wareth et al., 2019). As a result of its propensity to cause outbreaks of infections and develop resistance to most antibiotics, A. baumannii has become a serious health threat over the past few decades. Furthermore, 
clinical multidrug-resistant (MDR) A. baumannii isolates are gradually becoming prevalent globally (Magiorakos et al., 2012).

As a result of the lack of effective therapeutic drugs and new antibiotics, some traditional drugs such as polymyxins (colistin, polymyxin B), whose use has been discontinued, have had to be reinstated in the treatment of severe infections caused by MDR Gram-negative bacteria (Bassetti et al., 2013). MDR Gram-negative bacteria are highly sensitive to colistin. Thus, colistin is the last resort for the treatment of severe infections by Gram-negative pathogens, for example infections caused by MDR Pseudomonas aeruginosa, carbapenem-resistant Enterobacteriaceae, and MDR A. baumannii (Armengol et al., 2019). Unfortunately, with the increased use of colistin, colistinresistant strains have been detected. To ensure that this last barrier against MDR Gram-negative pathogens remains effective, appropriate action to delay the emergence of resistance and improve the efficacy of antibiotics is urgently needed. Antibiotic adjuvant therapy includes a combination of potent antibiotics and non-antibiotics, which interfere with the mechanism of antibiotic resistance or virulence (Trebosc et al., 2019). This may offer an alternative treatment strategy for infections caused by colistin-resistant $A$. baumannii.

Colistin resistance is conferred by phosphoethanolamine modification of the extracellular membrane, involving phosphoethanolamine transferase PmrC, EptA (Trebosc et al., 2019), and MCR-1 (Rolain et al., 2016). A limitation of colistin is that it can cause severe nephrotoxicity and neurotoxicity. Thus, it is often used in combination with other antibiotics to minimize the dose, and thereby limit its toxicity, while maximizing its antibacterial activity to prevent the emergence of drug resistance (Armengol et al., 2019). In recent years, the synergistic activity of colistin combined with other antibiotics against $A$. baumannii has been explored. Most studies have focused on its combination with carbapenems. The synergistic rate for polymyxin combined with meropenem and doripenem is reported to be approximately $85.0 \%$, which is higher than the synergistic rate with imipenem (66.8\%) (Ni et al., 2015). Dinc et al. (2015) studied the effect of colistin combined with doripenem in a mouse model of sepsis induced by carbapenem-resistant A. baumannii, and the effects of the combination therapy were superior to those of the single drug treatment group. In vivo experiments in a mouse model of XDR A. baumannii thigh infection demonstrated that the use of rifampicin significantly increased the efficacy of colistin, substantially reducing the bacterial load during infection in mice (Fan et al., 2016). Furthermore, the synergistic activity between polymyxin and glycopeptides, minocycline, sulbactam, and fosfomycin has also been reported (Claeys et al., 2014; Yang et al., 2016; Leelasupasri et al., 2018; Han et al., 2019).

Compared with synthetic antibiotics, phytochemicals have the characteristics of low toxicity and low cost, which make them a good choice as drugs or adjuvants. Consequently, phytochemicals are increasingly being used as alternative or complementary compounds in combination with conventional antibiotics (Shin et al., 2018). Capsaicin (8-methyl-N-vanillyl-6nonenamide) and related compounds (capsaicinoids) are
TABLE 1 | Antibiotic resistances of clinical A. baumannii strains Ab13 and Ab156. $\mathrm{R}$ represent resistance. $\mathrm{S}$ represent sensitive.

\begin{tabular}{lcc} 
Strain Antibiotics & Ab13 & Ab156 \\
\hline Piperacillin & $\mathrm{R}$ & $\mathrm{R}$ \\
Sulbactam & $\mathrm{R}$ & $\mathrm{R}$ \\
Tazobactam & $\mathrm{R}$ & $\mathrm{R}$ \\
Clavulanic acid & $\mathrm{R}$ & $\mathrm{R}$ \\
Ceftazidime & $\mathrm{R}$ & $\mathrm{R}$ \\
Cefepime & $\mathrm{R}$ & $\mathrm{R}$ \\
Cefotaxime & $\mathrm{R}$ & $\mathrm{R}$ \\
Imipenem & $\mathrm{R}$ & $\mathrm{R}$ \\
Meropenem & $\mathrm{R}$ & $\mathrm{R}$ \\
Gentamicin & $\mathrm{R}$ & $\mathrm{R}$ \\
Tobramycin & $\mathrm{R}$ & $\mathrm{R}$ \\
Amikacin & $\mathrm{R}$ & $\mathrm{R}$ \\
Doxycycline & $\mathrm{R}$ & $\mathrm{R}$ \\
Minocycline & $\mathrm{S}$ & $\mathrm{S}$ \\
Tetracycline & $\mathrm{R}$ & $\mathrm{R}$ \\
Ciprofloxacin & $\mathrm{R}$ & $\mathrm{R}$ \\
Levofloxacin & $\mathrm{S}$ & $\mathrm{R}$ \\
Gatifloxacin & $\mathrm{S}$ & $\mathrm{S}$ \\
Sulfisoxazole & $\mathrm{R}$ & $\mathrm{R}$ \\
Polymyxin B & $\mathrm{R}$ & $\mathrm{R}$ \\
& &
\end{tabular}

secondary metabolites of chili pepper, which play an important role in the immune defense of plants (Marini et al., 2015). Such compounds have important pharmacological and physiological properties such as anti-inflammatory, analgesic, anticancer, and cardioprotective properties, as well as exerting beneficial effects on the gastrointestinal system (Srinivasan, 2016). Additionally, capsaicin has attracted interest in recent years due to its antibacterial and antiviral activities. A recent study showed that capsaicin inhibits the growth of Vibrio cholerae and significantly reduces the expression of toxin-associated genes (Erfanimanesh et al., 2019). Another study demonstrated that capsaicin has an inhibitory effect on the growth and biofilm formation of Porphyromonas gingivalis, decreasing the secretion of gingivomucosal inflammatory cytokine (Zhou et al., 2014). To date, no reports have been published on the combination of colistin and capsaicin in the treatment of $A$. baumannii infection.

In our study, we detected the synergistic activity of colistin in combination with capsaicin against colistin-resistant $A$. baumannii in vitro and constructed a mouse bacteremia model to assess its in vivo activity. Mechanistic study demonstrated that capsaicin can inhibit the biofilm formation, decrease the production of intracellular ATP and disrupt the outer membrane of A. baumannii, but has no effect on the inner membrane. Transcription analysis shown that capsaicin can inhibits protein synthesis and efflux activity. Taken together, capsaicin dramatically rescued colistin efficacy on A. baumannii.

\section{MATERIALS AND METHODS}

\section{Reagents}

Colistin and capsaicin were obtained from Selleck (Huston, TX, United States) and Tocris Bioscience (Bristol, United Kingdom), respectively. For in vitro studies, capsaicin was dissolved in 
dimethyl sulfoxide (DMSO; Sigma-Aldrich, St. Louis, MO, United States), and then diluted with culture medium to the desired concentrations. For in vivo studies, capsaicin was dissolved in ethanol, and then diluted with normal saline to the required concentration, with a final ethanol concentration of 0.2\% (Zhang et al., 2018).

\section{Bacterial Strains and Susceptibility Test}

Two non-clonal MDR clinical A. baumannii strains Ab13 and Ab156 isolated at the Affiliated Hospital of Yangzhou University and the type strain ATCC 19606 were used in this study. All of these strains are sensitive to colistin. The distinct antibiotic resistance profiles of Ab13 and Ab156 are shown in Table 1. The minimum inhibitory concentrations (MICs) of colistin and capsaicin against $A$. baumannii were evaluated in triplicate, based on the standard broth microdilution method recommended by the Clinical and Laboratory Standards Institute (CLSI) (Matuschek et al., 2018). The criteria for the interpretation of the susceptibility tests were based on the CLSI guidelines.

\section{Induction of Colistin-Resistant $\boldsymbol{A}$. baumannii}

A. baumannii strains Ab13, Ab156, and ATCC 19606 were subcultured in $5 \mathrm{~mL}$ of Mueller-Hinton broth (MHB; Oxoid Ltd., Cambridge, United Kingdom) containing colistin (SigmaAldrich) at concentrations ranging from 0 to $32 \mu \mathrm{g} / \mathrm{mL}$ for $24 \mathrm{~h}$ at $37^{\circ} \mathrm{C}$ with shaking at $200 \mathrm{rpm}$. Resistant mutants were selected from $\mathrm{MH}$ agar plates containing $10 \mu \mathrm{g} / \mathrm{mL}$ of colistin and were then stored as glycerol stocks at $-80^{\circ} \mathrm{C}$. The obtained colistinresistant A. baumannii were designated Ab13-R, Ab156-R, and ATCC 19606-R, respectively.

\section{Checkerboard Assays}

Checkboard assays were carried out to test the MIC of the combination of colistin and capsaicin. In brief, increasing concentrations of capsaicin $(0-256 \mu \mathrm{g} / \mathrm{mL})$ in each column and increasing concentrations of colistin $(0-64 \mu \mathrm{g} / \mathrm{mL})$ in each row were set up in 96-well microtiter plates. Each well was inoculated with $100 \mu \mathrm{L}$ of suspension containing $5 \times 10^{5} \mathrm{CFU} /$ $\mathrm{mL}$ of the test strain, and then the required concentration of drugs was added to a final volume of $200 \mu \mathrm{L}$. The plates were incubated at $37^{\circ} \mathrm{C}$ for $24 \mathrm{~h}$. Data were obtained from at least three independent experiments.

\section{Time-Kill Assays}

The test strains were subjected to time-kill assays using colistin alone, capsaicin alone or colistin combination with capsaicin. Assays were performed in duplicate. Tubes containing MHB, with or without antibiotic, were inoculated with approximately $5 \times 10^{6} \mathrm{CFU} / \mathrm{mL}$ log-phase inoculum to a final volume of $5 \mathrm{~mL}$. Then, the tubes were cultured in an ambient atmosphere at $37^{\circ} \mathrm{C}$ with shaking at $200 \mathrm{rpm}$. The number of bacteria was determined at time points of $0,2,4,6,8$, and $12 \mathrm{~h}$ by enumerating colonies in 10-fold serially-diluted specimens of $100 \mathrm{~mL}$ aliquots plated on Mueller-Hinton agar at $37^{\circ} \mathrm{C}$. Synergism was defined as a $>2 \log 10$ decrease in $\mathrm{CFU} / \mathrm{mL}$ after $12 \mathrm{~h}$ when the combination treatment was used compared with colistin or capsaicin alone.

\section{Safety Assessment}

Hemolytic activity of colistin in the presence of capsaicin was evaluated as follows. $8 \%$ sheep blood cells that prepared from fresh sterile defibrinated sheep blood, were treated with various concentrations of colistin $(8-32 \mu \mathrm{g} / \mathrm{mL})$ alone or in combination with capsaicin $(8-128 \mu \mathrm{g} / \mathrm{mL})$ at $37^{\circ} \mathrm{C}$. After incubation for $1 \mathrm{~h}$, the absorption of supernatant was measured at $576 \mathrm{~nm}$ by Infinite M200 Microplate reader (Tecan). PBS (0.01M, pH = 7.4) was used as a negative control, and PBS with Triton X-100 (0.2\%) was used as a positive control. Comparing the absorbance of the sample with the positive control is the hemolysis rate.

Cytotoxicity on mammalian cells was performed on A549 cells (adenocarcinomic human alveolar basal epithelial cells)by Cell Counting Kit-8 (CCK-8) assay according to the manufacturer's instructions (Beyotime, Shanghai, China). A549 cells were seeded in a 96-well plate at about $2 \times 10^{3}$ cells/well and cultured for $20 \mathrm{~h}$. These cells were then treated with capsaicin $(8-128 \mu \mathrm{g} / \mathrm{mL})$ for $24 \mathrm{~h}$, with three replicates for each concentration. After $24 \mathrm{~h}$ of culture, $10 \mu \mathrm{l}$ of CCK- 8 solution per well was added. Absorbance was measured at $450 \mathrm{~nm}$ to calculate the cytotoxicity of capsaicin.

\section{Mouse Bacteremia Model}

For the in vivo experiments, the efficacy of colistin and capsaicin for the treatment of $A$. baumannii infection was determined using the murine bacteremia infection model. Four-week-old BALB/C male mice of approximately $20 \mathrm{~g}$, purchased from the Experimental Animal Center of Yangzhou University (Yangzhou, China), were infected intraperitoneally (i.p.) with a washed overnight culture of A. baumannii ATCC19606-R ( $1 \times$ $\left.10^{7} \mathrm{CFU}\right)$. After $24 \mathrm{~h}$, the mice were randomly divided into four groups ( $\mathrm{n}=6$ per group) and received daily treatment via i.p. injection: the control group received sterile PBS, the colistin group received $5 \mathrm{mg} / \mathrm{kg}$ colistin, the capsaicin group received $3 \mathrm{mg} / \mathrm{kg}$ capsaicin, and the combination group received $5 \mathrm{mg} / \mathrm{kg}$ colistin and $3 \mathrm{mg} / \mathrm{kg}$ capsaicin. Treatment lasted for 3 days. Twenty- 4 hours after the last therapeutic dose, mice were euthanized and the tissues (e.g., spleen, kidney, liver, and lungs) were removed, weighed, homogenized, and serially diluted in PBS. The bacterial counts in the target tissues of each group were computed and presented as the mean $( \pm \mathrm{SD})$ $\log 10 \mathrm{CFU} / \mathrm{g}$ tissue or $\log 10 \mathrm{CFU} / \mathrm{mL}$ blood.

Mice were handled following the Guidelines for the Care and Use of Laboratory Animals and procedures were approved by the Ethical Committee of Yangzhou University and the Laboratory Animal Management Committee of Yangzhou Province. All animals were acclimated to the controlled environment for 1 week prior to the experiment.

\section{Biofilm Formation and Quantification}

The effects of capsaicin on A. baumannii biofilm formation were analyzed using a 96-well tissue culture-treated polystyrene plate. The bacterial cell suspension $\left(1 \times 10^{6} \mathrm{CFU} / \mathrm{mL}\right)$ was cultured in wells supplemented with capsaicin $(0-128 \mu \mathrm{g} / \mathrm{mL})$. Negative controls containing $\mathrm{MH}$ only were included. The inoculated 
plates were incubated aerobically at $37^{\circ} \mathrm{C}$ for $24 \mathrm{~h}$. Then, the planktonic bacteria and remaining capsaicin were discarded and the wells were washed twice with phosphate-buffered saline (PBS) and fixed with paraformaldehyde (4\%) for $15 \mathrm{~min}$. After removing the paraformaldehyde and staining with $0.4 \%(\mathrm{w} / \mathrm{v})$ crystal violet (Sinopharm Chemical Reagent Co. Ltd., Shanghai, China) for $30 \mathrm{~min}$, the cells were finally washed with $\mathrm{ddH}_{2} \mathrm{O}$ until no color was visible in the control well. Aliquots of $95 \%$ ethanol were added to each well to dissolve the crystals. The average optical density values at $595 \mathrm{~nm}\left(\mathrm{OD}_{595}\right)$ were calculated for the samples. The rate of biofilm inhibition was calculated via the following equation (Wei et al., 2017):

[1-(OD 595 of cells treated with test agent/OD ${ }_{595}$ of untreated control) $] \times 100$.

The combination of colistin $(2 \mu \mathrm{g} / \mathrm{mL})$ and capsaicin $(32 \mu \mathrm{g} /$ $\mathrm{mL})$ or capsaicin $(32 \mu \mathrm{g} / \mathrm{mL})$ alone on the biofilm formation of colistin-resistant $A$. baumannii were done similar to the above study. Minimum biofilm inhibitory concentration (MBIC) is defined as the lowest concentration of an antimicrobial agent that can inhibit the formation of biofilms, and minimal biofilm eradication concentration (MBEC) is defined as the lowest concentration at which bacteria biofilms failed to regrow (Mi et al., 2016). The MBIC values of capsaicin on A. baumannii were done as the following steps: $100 \mu \mathrm{L}$ of bacterial suspensions $(1 \times$ $10^{6} \mathrm{CFU} / \mathrm{mL}$ ) were transferred to the wells of a flat-bottom 96well microtiter plate. Capsaicin at different concentrations $(0-512 \mu \mathrm{g} / \mathrm{mL})$ were then added and the plates were incubated at $37^{\circ} \mathrm{C}$ for $24 \mathrm{~h}$. Then, the consequent steps were done similar to the above study. The MBEC values of capsaicin on A. baumannii were the lowest concentration that can prevent visible growth in the biofilm recovery medium after $18 \mathrm{~h}$ incubation (Ceri et al., 1999; Lin et al., 2020).

\section{Biochemical Factors Measurement}

Outer membrane permeability:1-N-Phenyl-naphthylamine (NPN) assay was used to assess the outer membrane permeability according to a previously study (Jangra et al., 2018). Due to NPN is a hydrophobic fluorescent probe, the fluorescence of NPN increases when it is transferred from aqueous to hydrophobic environment. The permeability of NPN were tested using 96 well plates (black with clear bottom). After treating the bacteria with different concentrations of capsaicin $(0-128 \mu \mathrm{g} / \mathrm{mL})$ or colistin $(0-32 \mu \mathrm{g} / \mathrm{mL})$ without or with capsaicin $(32 \mu \mathrm{g} / \mathrm{mL})$ for $1 \mathrm{~h}, 1$ $\mathrm{N}$-phenylnaphthylamine (NPN) was added with a final concentration of $10 \mu \mathrm{M}$, and the fluorescence intensity were measured with the excitation wavelength of $350 \mathrm{~nm}$ and emission wavelength of $420 \mathrm{~nm}$.

Cell membrane integrity:Cell membrane integrity experiments were performed as previously described with some modifications (Jangra et al., 2019). Propidium iodide (PI, Thermo Fisher Scientific), a membrane-impermeable fluorescence dye, was used to detect the cell membrane integrity. After washing bacterial cells three times with normal saline $(0.9 \% \mathrm{NaCl})$, the cell concentration was normalized to an $\mathrm{OD}_{600}$ of 0.1 . Then, treat the bacteria cells with different concentrations of capsaicin $(0-128 \mu \mathrm{g} / \mathrm{mL})$ or colistin $(0-32 \mu \mathrm{g} / \mathrm{mL})$ without or with capsaicin $(32 \mu \mathrm{g} / \mathrm{mL})$ for $1 \mathrm{~h}$, the supernatants were collected and PI was added to a final concentration of $10 \mathrm{nM}$. The fluorescence intensity was measured with the excitation wavelength of $535 \mathrm{~nm}$ and emission wavelength of $615 \mathrm{~nm}$.

Total ROS: The levels of reactive oxygen species (ROS) in $A$. baumannii were measured using $2^{\prime}, 7^{\prime}$-dichlorodihydrofluorescein diacetate (DCFH-DA) according to the manufacturer's instructions (Beyotime, Shanghai, China). With the entering of DCFH-DA into A. baumannii cells, DCFH-DA was switched to a membraneimpermeable homologous by cellular esterases or oxidized to a fluorescent form by hydroxyl radical, superoxide, orhydrogen peroxide $\left(\mathrm{H}_{2} \mathrm{O}_{2}\right)$ (Chen et al., 2020). Resistant strains were loaded with DCFH-DA at a final concentration of $10 \mu \mathrm{M}$, and then treated by different concentrations of capsaicin $(0-128 \mu \mathrm{g} / \mathrm{mL})$. Fluorescence intensity was measured with the excitation wavelength of $488 \mathrm{~nm}$ and emission wavelength of $525 \mathrm{~nm}$.

Intracellular ATP: Intracellular ATP levels of A. baumannii were determined using an Enhanced ATP Assay Kit (Beyotime, Shanghai, China). The A. baumannii were cultured overnight and resuspended in PBS, then treated with different concentrations of capsaicin $(0-128 \mu \mathrm{g} / \mathrm{mL})$ for $1 \mathrm{~h}$. After centrifugation, the bacterial precipitates were treated with lysozyme and the supernatants were collected for subsequent determination. The test solutions were added into the 96-well plates and incubated at room temperature for $5 \mathrm{~min}$. The luminescence was detected by multi-function microplate reader (Biotek, United States).

\section{Scanning Electron Microscopy}

ATCC19606-R were treated with colistin $(2 \mu \mathrm{g} / \mathrm{mL})$ or capsaicin $(32 \mu \mathrm{g} / \mathrm{mL})$ or their combination for $4 \mathrm{~h}$ and were fixed with $2.5 \%$ glutaraldehyde at $4^{\circ} \mathrm{C}$ for $24 \mathrm{~h}$. Then the bacteria were gradual dehydration with ethanol $(30,50,70,80,90,95$, and $100 \%)$. The processed samples were dried using a critical point dryer and coated with a layer of gold-palladium using an ion sprayer, and finally observed with SEM (GeminiSEM 300).

\section{Transcriptomic Analysis}

ATCC19606-R were grown in MHB to the early exponential phase, and treated with colistin $(2 \mu \mathrm{g} / \mathrm{mL})$ alone or in combination of capsaicin $(32 \mu \mathrm{g} / \mathrm{mL})$ for $4 \mathrm{~h}$. After quality inspection of RNA samples, cDNA synthesis, cDNA fragmentation modification and sorting, and library amplification were processed, then, the constructed cDNA library was sequenced using Illumina HiSeqTM. The technical services of this process and subsequent bioinformatics analysis were provided by Nanjing Motif Zhigu Biotechnology Co., Ltd.

\section{Statistical Analysis}

Data were presented as mean \pm standard deviation (SD) of three replications. Mean values between groups were compared using the two-tailed Student's t-test. All statistical analyses were performed using GraphPad Prism version 5.04 (GraphPad Software Inc., San Diego, CA, United States) with a $p$ value < 0.05 considered significant. 
TABLE 2 | Colistin MICs of wild-type and laboratory-generated colistin-resistant strains when treated with different concentrations of capsaicin.

Strain
Colistin MIC $(\mu \mathrm{g} / \mathrm{mL})$ at different Capsaicin concentrations $(\mu \mathrm{g} / \mathrm{mL})$

\begin{tabular}{|c|c|c|c|c|c|c|c|c|c|c|}
\hline \multirow{2}{*}{$\begin{array}{c}\text { Only } \\
\text { colistin }\end{array}$} & \multicolumn{2}{|c|}{ Capsaicin (256) } & \multicolumn{2}{|c|}{ Capsaicin (128) } & \multicolumn{2}{|c|}{ Capsaicin (64) } & \multicolumn{2}{|c|}{ Capsaicin (32) } & \multicolumn{2}{|c|}{ Capsaicin (16) } \\
\hline & MIC & $\begin{array}{c}\text { Fold } \\
\text { change }\end{array}$ & MIC & $\begin{array}{c}\text { Fold } \\
\text { change }\end{array}$ & MIC & $\begin{array}{c}\text { Fold } \\
\text { change }\end{array}$ & MIC & $\begin{array}{c}\text { Fold } \\
\text { change }\end{array}$ & MIC & $\begin{array}{c}\text { Fold } \\
\text { change }\end{array}$ \\
\hline 0.5 & - & - & - & - & - & - & - & - & - & - \\
\hline 1,024 & 0.5 & 2048 & 0.5 & 2048 & 0.5 & 2048 & 0.5 & 2048 & 8 & 128 \\
\hline 1 & - & - & - & - & - & - & - & - & - & - \\
\hline 128 & 1 & 128 & 4 & 32 & 4 & 32 & 4 & 32 & 16 & 8 \\
\hline 2 & - & - & - & - & - & - & - & - & - & - \\
\hline 64 & 0.5 & 128 & 0.5 & 128 & 0.5 & 128 & 0.5 & 128 & 4 & 16 \\
\hline
\end{tabular}
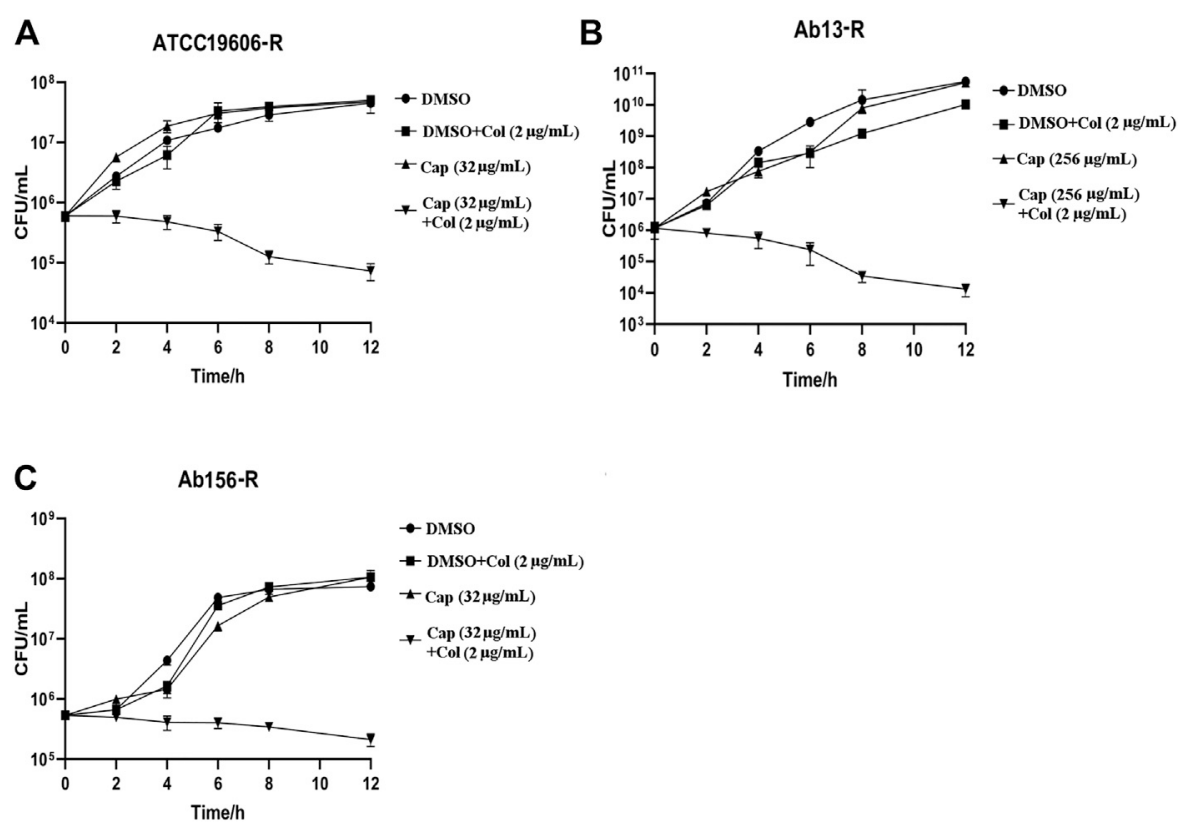

FIGURE 1 | Time-kill curves of A. baumannii isolates using the drugs alone or in combination. The capsaicin concentrations of ATCC19606-R, Ab13-R and Ab156R were $32 \mu \mathrm{g} / \mathrm{mL}, 256 \mu \mathrm{g} / \mathrm{mL}, 32 \mu \mathrm{g} / \mathrm{mL}$, respectively; at which the MIC values of colistin are below the susceptibility breakpoint (2 $\mu \mathrm{g} / \mathrm{mL}$ ). (A): Time-kill curves of ATCC19606-R using capsaicin (32 $\mathrm{gg} / \mathrm{mL})$ or colistin $(2 \mu \mathrm{g} / \mathrm{mL})$ without or with capsaicin $(32 \mu \mathrm{g} / \mathrm{mL})$. (B): Time-kill curves of Ab13-R using capsaicin (256 $\mu \mathrm{g} / \mathrm{mL})$ or colistin $(2 \mu \mathrm{g} / \mathrm{mL})$ without or with capsaicin $(256 \mu \mathrm{g} / \mathrm{mL})$. (C): Time-kill curves of Ab156-R using capsaicin (32 $\mu \mathrm{g} / \mathrm{mL}) \mathrm{or}$ colistin $(2 \mu \mathrm{g} / \mathrm{mL})$ without or with capsaicin (32 $\mu \mathrm{g} / \mathrm{mL})$. Abbreviations: Cap, capsaicin; Col, colistin; DMSO, dimethyl sulfoxide.

\section{RESULTS}

\section{Synergistic Activity of Colistin in Combination With Capsaicin in a Checkerboard Assay}

The potency of capsaicin as an antibacterial was initially evaluated against colistin-resistant strains using standard broth dilution assays to determine MICs. The MICs of capsaicin were $>512 \mu \mathrm{g} / \mathrm{mL}$ for all of the strains tested, revealing the lack of direct antimicrobial activity of capsaicin alone against the ATCC 19606$\mathrm{R}, \mathrm{Ab13}-\mathrm{R}$, and Ab156-R colistin-resistant strains.

To explore the interaction of capsaicin and colistin, a checkerboard assay was performed to determine the FIC indices for the laboratorygenerated colistin-resistant $A$. baumannii. Checkerboard assays revealed the obvious dose-dependent synergistic activity of capsaicin with colistin on the ATCC 19606-R (FICI = 0.03), Ab13-R (FICI = 0.06), and Ab156-R (FICI = 0.04) colistinresistant strains (Table 2). Capsaicin could decrease the colistin MICs to values lower than the susceptibility breakpoint $(2 \mu \mathrm{g} / \mathrm{mL})$ in most cases (Table 2). These results suggested that colistin-resistant A. baumannii strains have recovered or enhanced sensitivity to colistin when combined with different concentrations of capsaicin.

\section{Synergistic Activity of Capsaicin and Colistin in Time-Kill Assays}

To further confirm the synergism of capsaicin with colistin, timekill assays were carried out with the three colistin-resistant $A$. baumannii strains. The colistin concentration used corresponded 

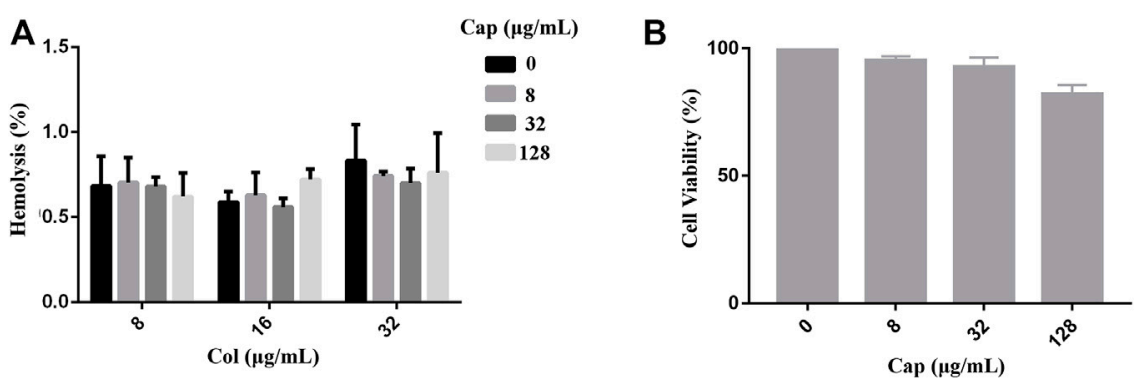

FIGURE 2 | Safety assessment of capsaicin. (A): Hemolytic activity of capsaicin (8-128 $\mu \mathrm{g} / \mathrm{mL})$ in the absence or presence of colistin (8-32 $\mu \mathrm{g} / \mathrm{mL})$ to the RBCs.

(B): In vitro mammalian toxicity in A549 cell lines. The data shown here are mean \pm SD of three replicates.
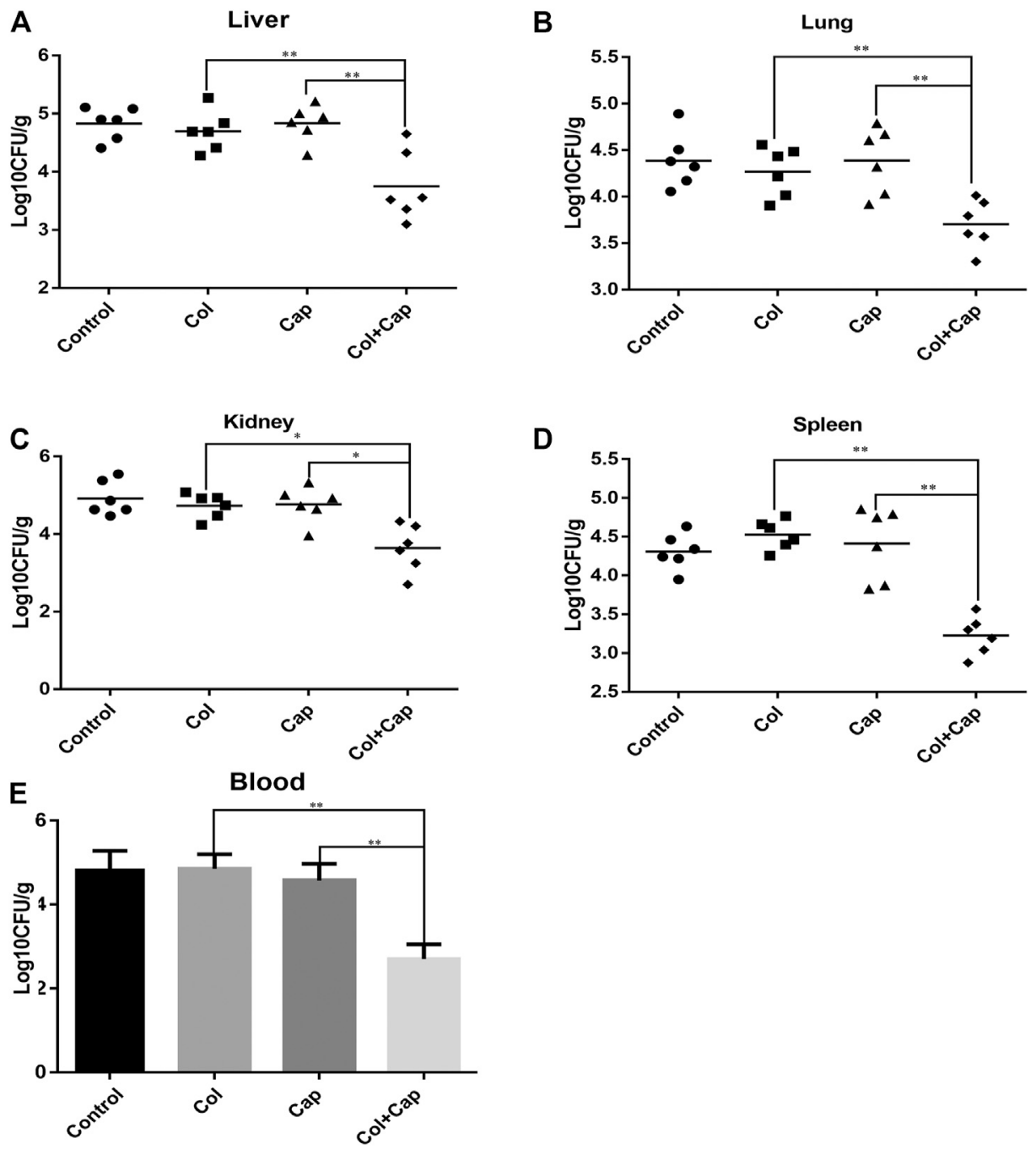

FIGURE 3 $\mid$ A. baumannii burden in the target tissues [(A): liver, (B): lung, (C): kidney, (D): spleen] were quantified as CFU per gram of target tissue in the murine bacteremia model following different regimens. E: Number of $A$. baumannii contained within the blood. The control group received sterile PBS, the colistin group received $5 \mathrm{mg} / \mathrm{kg}$ colistin, the capsaicin group received $3 \mathrm{mg} / \mathrm{kg}$ capsaicin, and the combination group received $5 \mathrm{mg} / \mathrm{kg}$ colistin and $3 \mathrm{mg} / \mathrm{kg}$ capsaicin. ${ }^{\star \star} \mathrm{p}<0.01 \mathrm{was}$ determined using the Student's t-test. At least six mice per group were analyzed. 

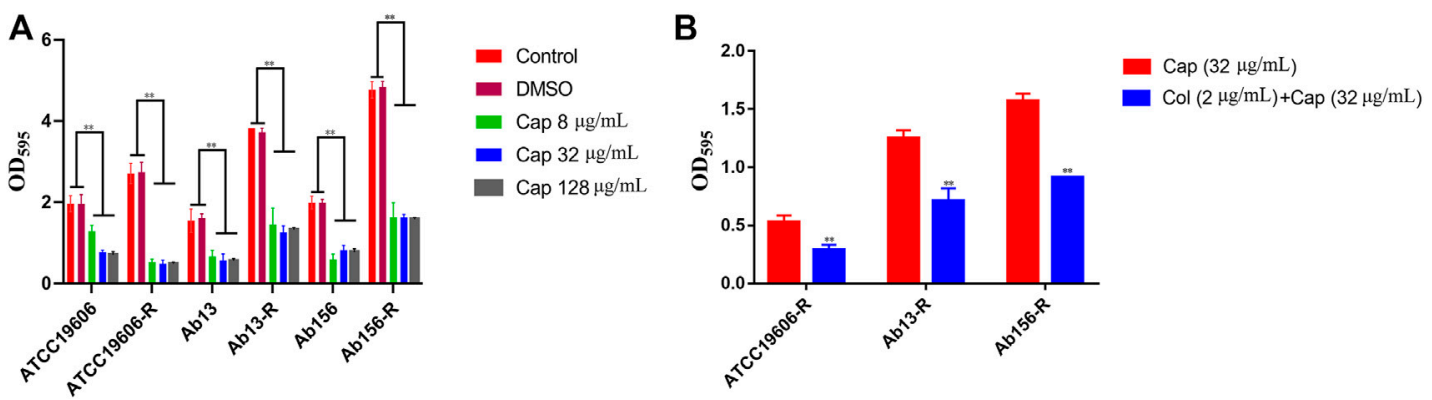

FIGURE 4 | (A): Effect of capsaicin (0-128 $\mu \mathrm{g} / \mathrm{mL})$ on biofilm formation of $A$. baumannii. (B): The combination of colistin $(2 \mu \mathrm{g} / \mathrm{mL})$ and capsaicin (32 $\mu \mathrm{g} / \mathrm{mL})$ on the biofilm formation of $A$. baumannii. ${ }^{\star} p<0.05,{ }^{\star \star} p<0.01$, data are expressed as the mean \pm SD of three replicates.

to the clinical breakpoint for susceptibility $(2 \mu \mathrm{g} / \mathrm{mL})$, and the capsaicin concentration used for Ab13-R was $256 \mu \mathrm{g} / \mathrm{mL}$, while that used for Ab156-R and ATCC19606-R was $32 \mu \mathrm{g} / \mathrm{mL}$, which could inhibit the growth of most bacteria when combined with colistin (Table 2). As shown in Figure $1 \mathrm{~A}, \mathrm{~B}$, and $\mathrm{C}$, three colistin-resistant strains grew normally within $12 \mathrm{~h}$ of using the drug alone. Neither capsaicin nor colistin monotreatment killed colistin-resistant A. baumannii. However, the combination led to a reduction of bacterial load approximate $>2 \log 10$ (Figure 1). The above results showed that capsaicin indeed drastically enhanced the effect of colistin against colistin-resistant A. baumannii.

\section{Safety Evaluation of Capsaicin}

To evaluate the safety of capsaicin, we performed toxicity tests in red blood cells (RBCs) and mammalian cells. As shown in Figure 2 A, we found that capsaicin $(\leq 128 \mu \mathrm{g} / \mathrm{mL})$ had negligible effects on the hemolysis of colistin to red blood cells (RBCs). The results of CCK-8 assay shown that capsaicin $(\leq 32 \mu \mathrm{g} / \mathrm{mL})$ had no cytotoxicity to A549 cells (Figure 2B). $128 \mu \mathrm{g} / \mathrm{mL}$ capsaicin has a lower cell viability of $82 \%$ (Figure 2 B), which is different with the results of hemolytic activity. Thus, the use of capsaicin is relatively safe under the concentrations of $128 \mu \mathrm{g} / \mathrm{mL}$.

\section{Therapeutic Efficacy of Colistin in Combination With Capsaicin in the Murine Model of Bacteremia}

Lethal bacteremia is a common outcome of $A$. baumannii infection so to determine the potential use of combination therapy in clinical situations, we investigated the in vivo efficacy of capsaicin combined with colistin in a bacteremia model infected with colistin-resistant $A$. baumannii. In preliminary experiments, we found that, by $24 \mathrm{~h}$, all organs in the mice were heavily infected with A. baumannii, suggesting that the infection was systemic. Furthermore, the combined treatment group showed reduced $A$. baumannii densities in the target tissues compared with the untreated or COL/CAP alone groups (Figure 3).

\section{Effect of Capsaicin on Biofilm Formation}

All of the strains used in this study have the ability to form biofilm. Considering the synergistic effects of capsaicin on colistin observed above, we hypothesized that capsaicin might inhibit biofilm formation of $A$. baumannii and this was tested using 8,32 and $128 \mu \mathrm{g} / \mathrm{mL}$ of capsaicin. As shown in Figure 4, capsaicin could suppress the biofilm formation of all test strains. Interestingly, the amount of biofilms formed by colistin-resistant strain ATCC19606-R, Ab13-R, and Ab156-R were significantly increased compared with corresponding sensitive strains, suggesting that colistin resistance was positively correlated with biofilm formation in this strain. This provides insight into the potential coevolution of antimicrobial resistance and biofilm formation in Gram-negative pathogens. The MBIC values of capsaicin on ATCC19606, ATCC19606-R, Ab13 and Ab156 were $256 \mu \mathrm{g} / \mathrm{mL}$. The MBIC values of capsaicin on Ab13-R and Ab156-R were $512 \mu \mathrm{g} / \mathrm{mL}$. The MBEC values of capsaicin on ATCC19606, ATCC19606-R, Ab13 and Ab156 were $512 \mu \mathrm{g} / \mathrm{mL}$, and the MBEC values of capsaicin on Ab13-R and Ab156-R were higher than $512 \mu \mathrm{g} / \mathrm{mL}$. The MBIC and MBEC values of ATCC19606, ATCC19606-R, Ab13 and Ab156 were lower than Ab13-R and Ab156-R. This could be due to the differences in the ability to form biofilms of the bacterial strains tested.

\section{Capsaicin Destroys the Outer Membrane}

We further measured the changes of cell membrane permeability after capsaicin treatment. NPN (1-N-phenylnaphthylamine) was used to study the outer membrane permeability of three resistant strains after capsaicin treatment. Due to the experiments calibrated in the same way, the fluorescence intensities showed in Figure $5 \mathrm{~A}$ and $\mathrm{B}$ were comparable. Figures 5A,B showed that the addition of capsaicin significantly increased outer membrane permeability in a concentration dependent manner. PI (propidium iodide) is often used to evaluate the integrity of inner membrane. As shown in Figures $\mathbf{5 C , D}$, there was no significant changes in fluorescence intensity among the three resistant strains exposed to capsaicin alone or combination with colistin. Similarly, the fluorescence intensities showed in Figures 5C,D were also comparable. These results indicated that capsaicin destroyed the integrity of outer membrane, thus increasing the membrane-damaging ability of colistin, but had no effect on the integrity of inner membrane.

\section{Capsaicin Reduces Intracellular ATP Content}

The effect of capsaicin on intracellular ATP level was determined by luciferase bioluminescence method. As shown in Figure 6A, capsaicin significantly decreased intracellular ATP level in a dose-dependent 


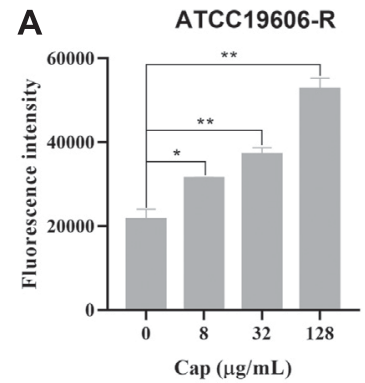

B

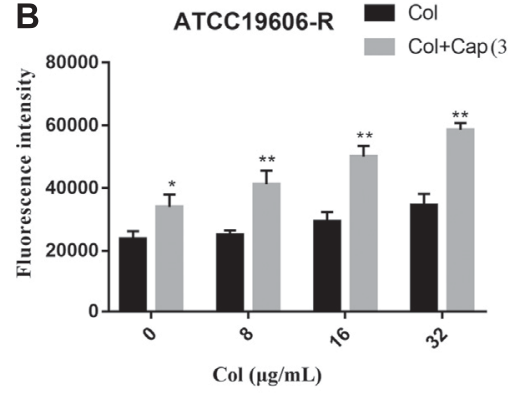

C

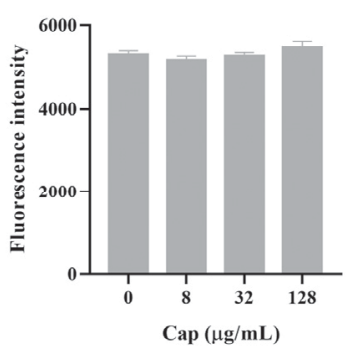

D

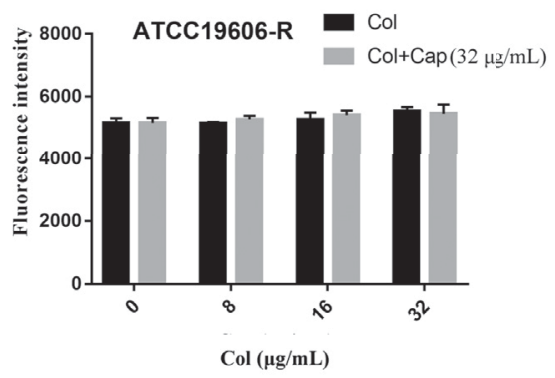

Ab13-R

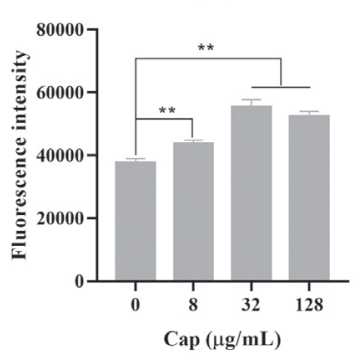

Ab13-R Col

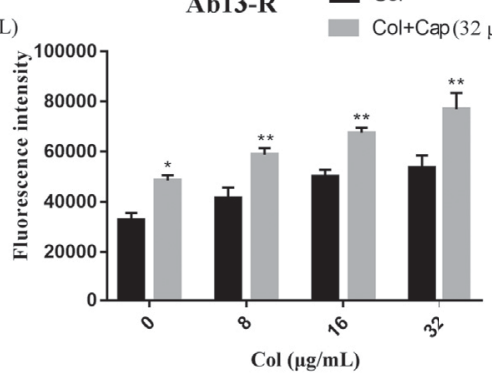

Ab13-R
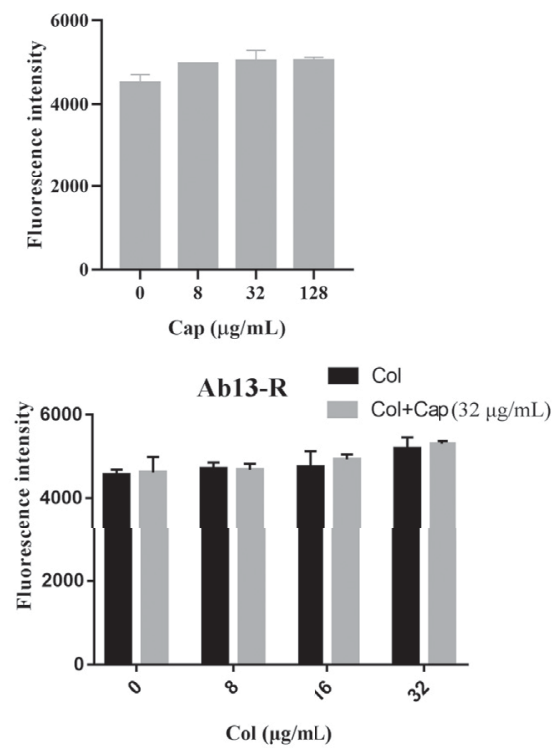

Ab156-R

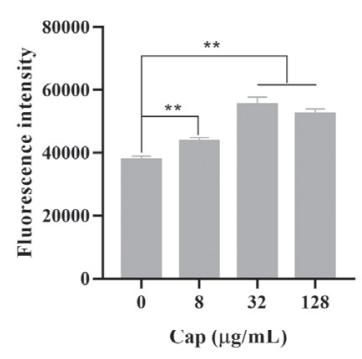

Ab156-R Col

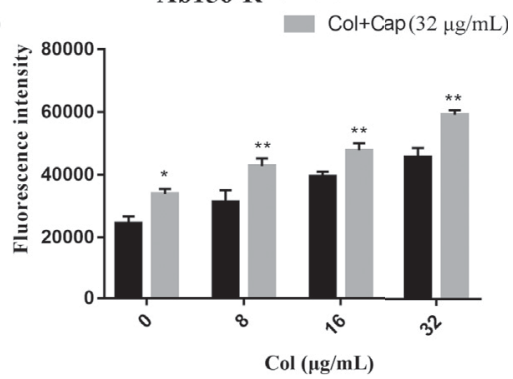

Ab156-R
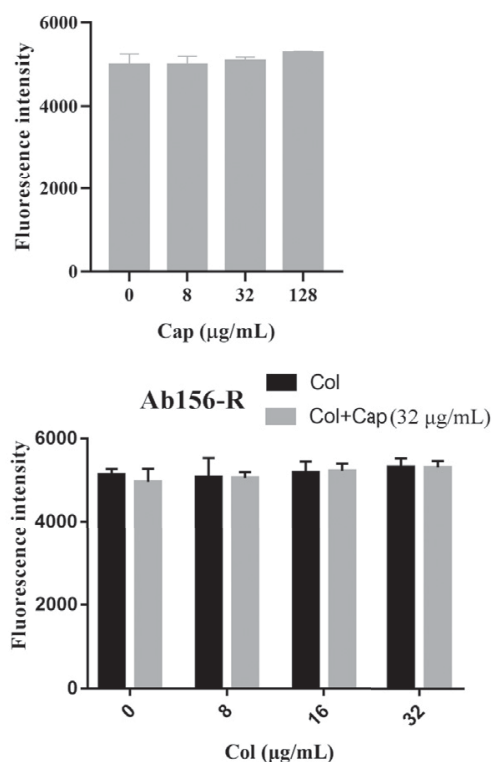

FIGURE 5 |Effect of capsaicin on the permeability of bacterial membrane. (A, B): Capsaicin disrupts the outer membrane of $A$. baumannii ATCC19606-R, AB13-R and AB156-R by measuring fluorescence intensity of 1-N-phenylnaphthylamine (NPN).(C, D): No effect on membrane permeability for propidium iodide (PI) in $A$. baumannii ATCC19606-R, AB13-R and AB156-R after treatment with capsaicin. Bacteria cells were treated with different concentrations of capsaicin (0-128 $\mu \mathrm{g} / \mathrm{mL})$ or colistin $(0-32 \mu \mathrm{g} / \mathrm{mL})$ without or with capsaicin $(32 \mu \mathrm{g} / \mathrm{mL})$.

manner. We speculated that the decrease of intracellular ATP level is related to the increase bactericidal effect of colistin.

\section{The Antioxidant Effect of Capsaicin}

The membrane damage is related to the production of ROS and affects the content of ATP, and the bacteria will produce oxidative damage under antibiotic stress. Therefore, we speculate that capsaicin may increase oxidative damages. As shown in Figure 6B, capsaicin decreased the ROS levels of ATCC19606-
$\mathrm{R}$ and Ab13-R, while in Ab156-R strain, capsaicin had no effect on ROS levels. It shows that capsaicin mainly plays its antioxidant activity here, though there are differences in different strains.

\section{The Morphological Changes of $\boldsymbol{A}$. baumannii Under Capsaicin Treatment}

The resistance of colistin is mainly mediated by the decrease of affinity between colistin and LPS. Therefore, we speculate that 


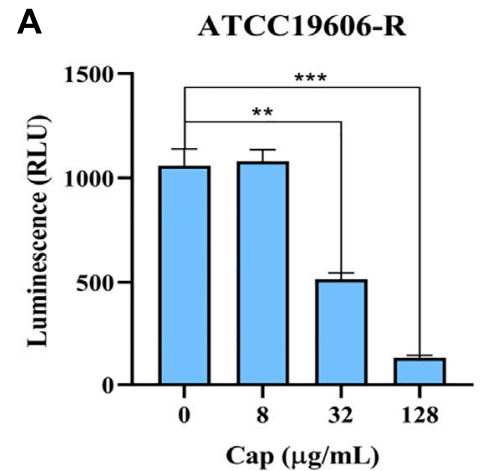

B

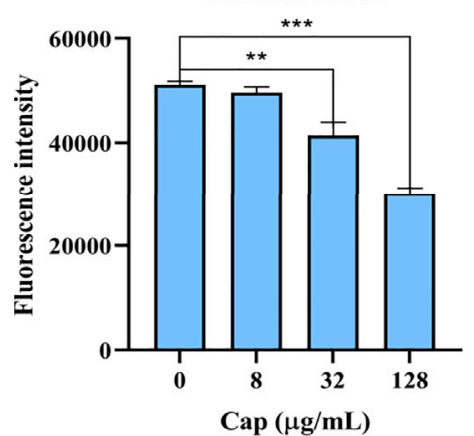

Ab13-R

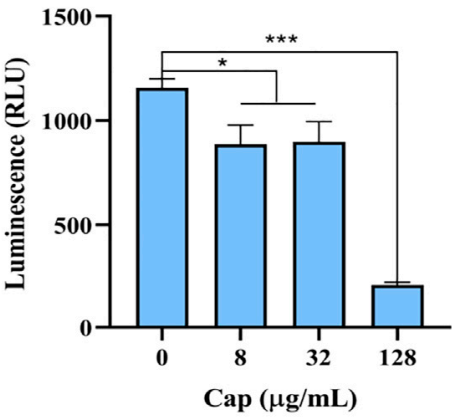

Ab13-R

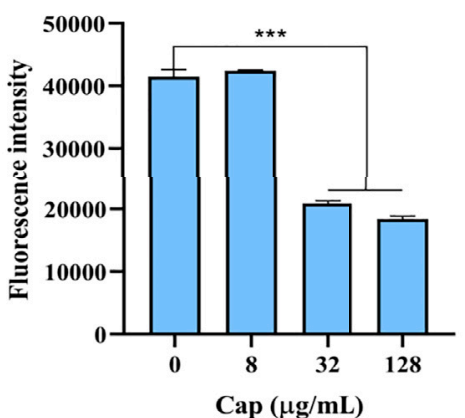

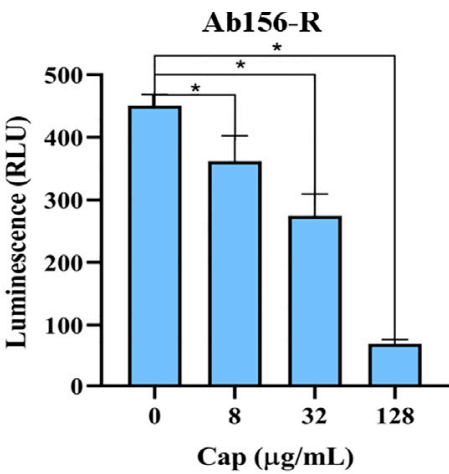

Ab156-R

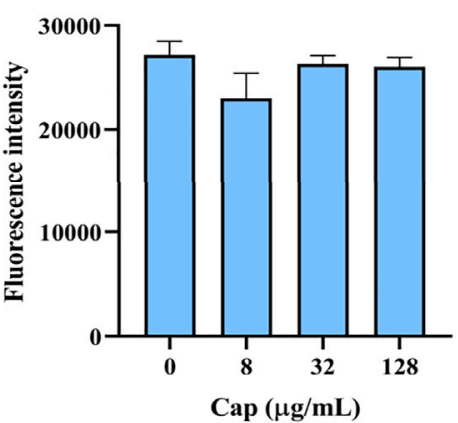

FIGURE 6 | (A): Intracellular ATP in bacterial cells treated with increasing concentrations of capsaicin $(0-128 \mu \mathrm{g} / \mathrm{mL})$. (B): The changes of total ROS of $A$. baumannii ATCC19606-R, AB13-R and AB156-R detected by DCFH-DA fluorescent probe after treatment with capsaicin (0-128 $\mu \mathrm{g} / \mathrm{mL})$.

capsaicin can restore the ability of colistin to destroy the outer membrane. In order to verify this hypothesis, scanning electron microscopy (SEM) was used to observe the morphological changes of ATCC19606-R after capsaicin treatment. As shown in Figure 7, the surface of cells treated colistin combination with capsaicin showed depression, shrinkage and even collapse and lysis compared with monotreatment, indicating that the bactericidal effect of capsaicin may be related to the rapid destruction of cell surface structure.

\section{Capsaicin Inhibits Protein Synthesis and Efflux Activity}

To gain a deeper understanding of the synergy mechanisms of capsaicin, we performed transcription analysis of ATCC19606-R after exposure to colistin or colistin-capsaicin combination for $4 \mathrm{~h}$. The results showed that compared with colistin alone group, the expression of 271 genes in ATCC19606-R were up-regulated and 327 genes were down regulated after $4 \mathrm{~h}$ treatment with capsaicin and colistin (Figure 8A). KEGG enrichment analysis showed that the up-regulated genes were significantly enriched in ribosome synthesis (Figure 8B), while the down regulated genes were involved in microbial metabolism (Figure 8C). In addition, the $30 \mathrm{~S}$ and $50 \mathrm{~S}$ subunit synthesis-related genes were upregulated under the combined treatment, which may be due to the inhibition of protein synthesis by capsaicin that was compensated by the up-regulation of ribosomal synthesis genes. The expression of multidrug efflux related genes were significantly down regulated, which indicated that the efflux pumps function of bacteria were inhibited under combined treatment. In addition, the expression of outer membrane protein related-genes also showed decreased, and these proteins were related to the ability of adhesion, invasion, colonization and proliferation (Figure 8D).

\section{DISCUSSION}

Acinetobacter spp. are glucose-non-fermentative, aerobic, Gramnegative coccobacilli. The ability of $A$. baumannii to form biofilms on the surface of inanimate objects enables $A$. baumannii to survive in hospital environments, and frequently cause pneumonia and septicemia in immunocompromised patients. A. baumannii possesses chromosomal genetic elements that confer resistance against many classes of antibiotics, as well as allowing for survival on inanimate surfaces for prolonged periods of time and strong tolerance to desiccation (Pourhajibagher et al., 2016). Carbapenem-associated MDR A. baumannii have been increasingly reported, and the treatment options for infections caused by this pathogen are severely limited. Colistin is currently one of the few antibiotics that remains active against this pathogen, and is considered the last resort treatment for severe Gram-negative infections (Kaye et al., 2016). However, treatment failures with colistin 

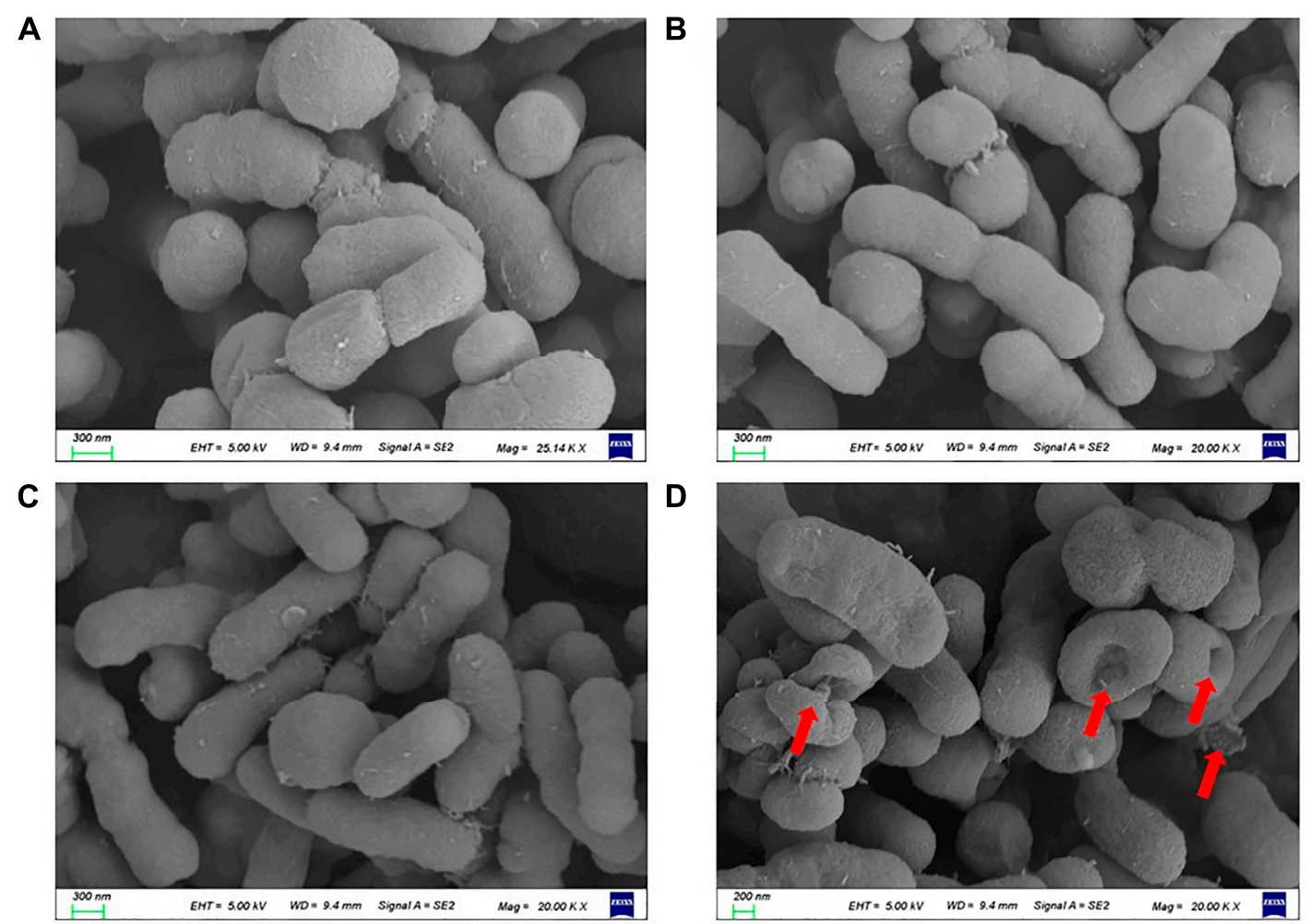

FIGURE 7 | Morphological changes of ATCC19606-R after different treatments. (A): Untreated. (B): Colistin (2 $\mu \mathrm{g} / \mathrm{mL})$ alone treatment. (C): Capsaicin (32 $\mu \mathrm{g} / \mathrm{mL})$ alone treatment. (D): Colistin $(2 \mu \mathrm{g} / \mathrm{mL})$ combined with capsaicin $(32 \mu \mathrm{g} / \mathrm{mL})$.

monotherapy and the emergence of drug resistance have promoted the search for combination therapy that synergistically kills colistin susceptible and resistant organisms (Thomas et al., 2019). A promising alternative strategy is provided by non-antibiotic drugs that can overcome resistance mechanisms when combined with failing antibiotics (Brown, 2015).

Many alternative therapies have been studied and amongst these, medicinal plants with various antimicrobial properties, have received significant attention. Furthermore, there are few reports of antimicrobial resistance to these phytochemicals, possibly because of the diversity of their mechanisms of action. In fact, quinones, tannins, terpenoids, alkaloids, flavonoids, and polyphenols are all valuable secondary metabolites that are used by plants in the defense against predation by herbivorous insects and microorganisms (Subramani et al., 2017). The antimicrobial activity and mechanisms of action of these plant-derived compounds have been reported, and they exert their effects through virulence factors that are critical for pathogenicity (Upadhyay et al., 2014).

Capsaicin, found in capsicum fruits, is the predominant, naturally-occurring alkamide (Basith et al., 2016). Capsaicin has a wide range of biological activities, which include antioxidant, analgesic, antimicrobial, and anticancer activities, as well as acting against high cholesterol levels and obesity. In this study, we demonstrated that capsaicin can function as an antibiotic adjuvant in vitro and in vivo, potentiating colistin activity against colistin-resistant A. baumannii. Because of the high rates of antibiotic resistance and the resulting treatment failures, $A$. baumannii bacteremia is becoming increasingly challenging to treat and is associated with a high mortality rate (Xiong et al., 2019). In the murine model of bacteremia, capsaicin-colistin combination therapy significantly reduced the bacterial load in the target tissues compared with the untreated control and the colistin/capsaicin monotherapy groups.

A. baumannii clinical isolates possess a strong ability to form biofilms. Biofilms are formed when microbial cells become surrounded by self-produced exopolysaccharide matrices on the surfaces of biotic or abiotic surfaces (Eze et al., 2018). Within a biofilm community, A. baumannii is more tolerant to extracellular stressors (Harding et al., 2018); hence, higher doses of antibiotics are needed to treat an infection involving biofilm than for planktonic cells, with the resulting risk of antibiotic resistance leading to increased treatment failures. In this study, we found that capsaicin has a significant inhibitory effect on the biofilms of both sensitive and resistant strains, which was consistent with a previous report by Zhou et al. (2014). Therefore, we supposed that the synergistic effect of capsaicin on colistin is partly due to its inhibition of biofilm, which weakens the aggregation of bacterial cells and alleviates the barrier of drug diffusion, enhancing the antibacterial effect of colistin.

The plant antimicrobial agents play a synergistic role in a variety of ways, which are crucial to the normal cell function or virulence. Although the exact mechanisms of antibacterial action of plant antimicrobial agents are still unclear, some potential mechanisms 
A
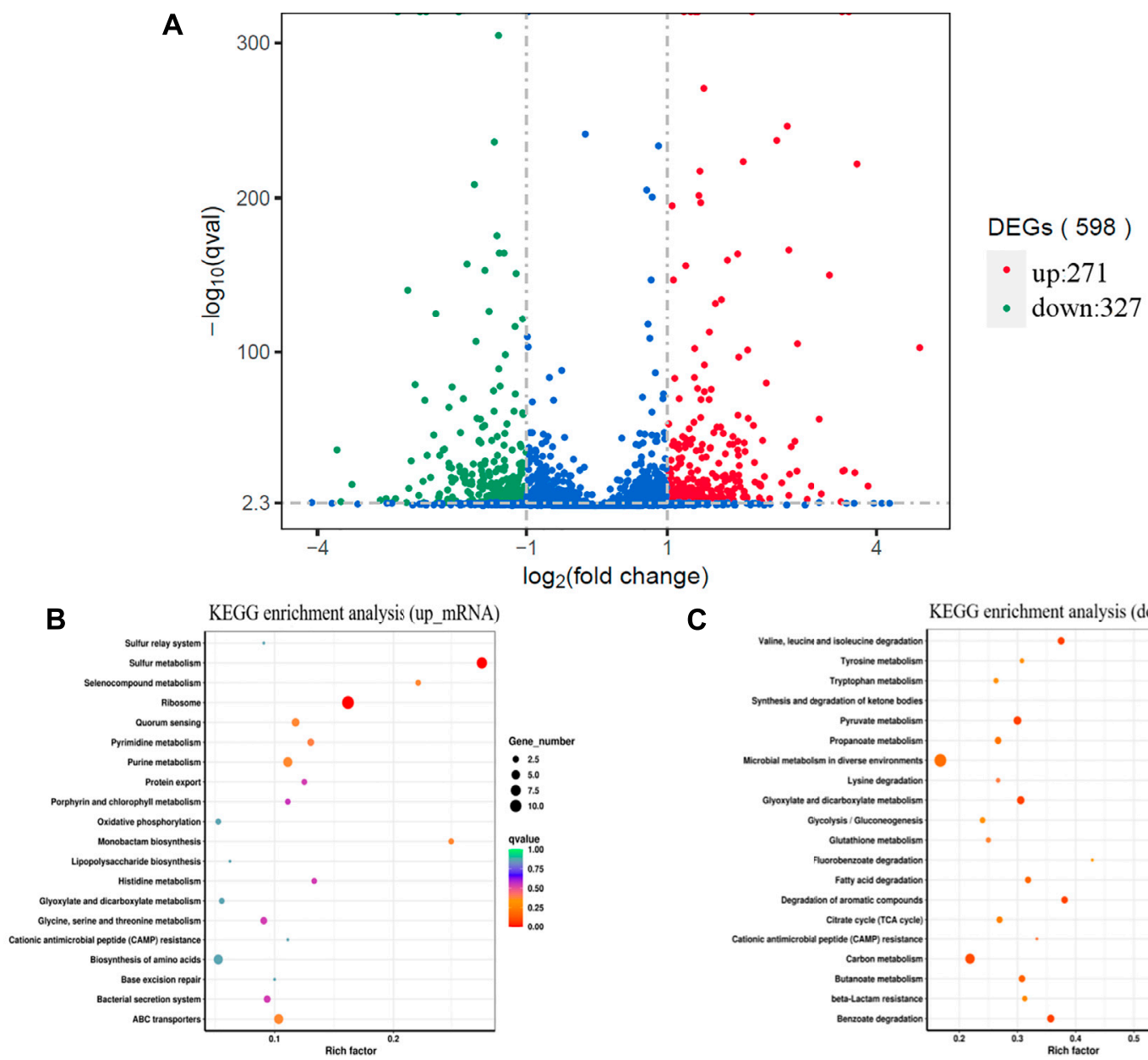

C

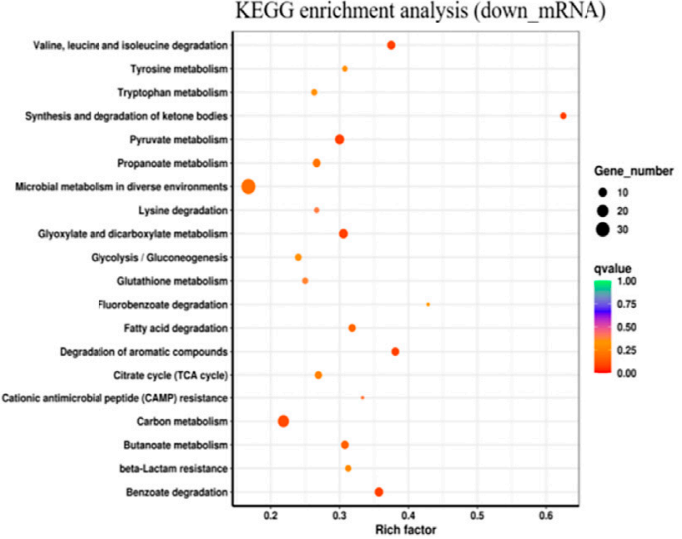

D

Col

Col+Cap Log2FC

Col

Col+Cap Log2FC

Col

Col+Cap Log2FC
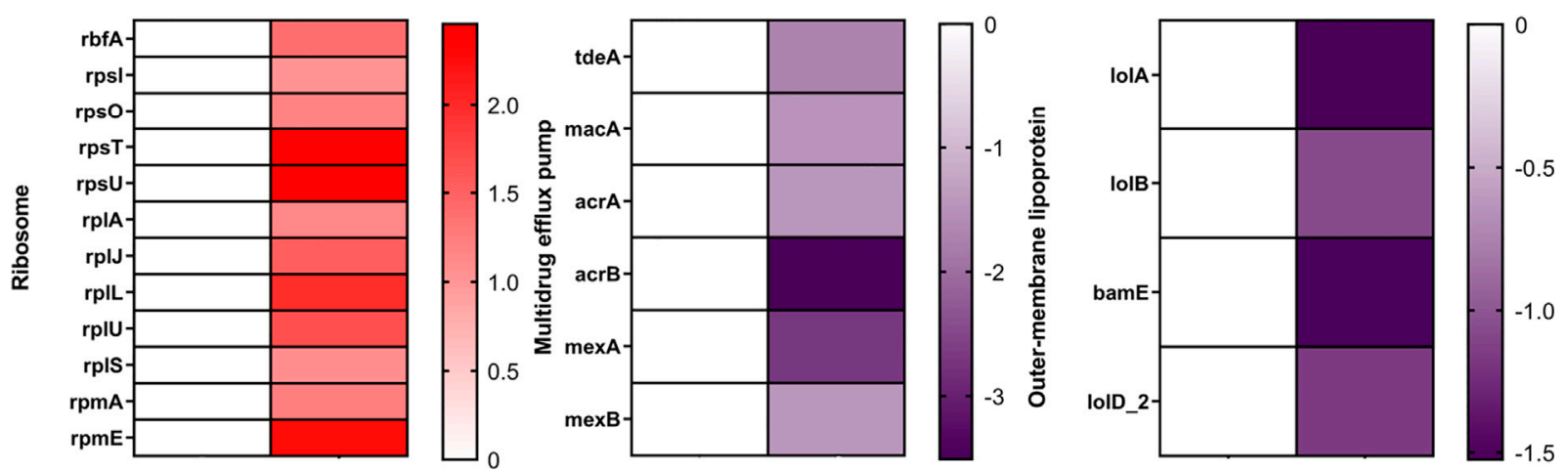

FIGURE 8 | Transcriptome analysis of ATCC19606-R after exposure to colistin $(2 \mu \mathrm{g} / \mathrm{mL})$ alone or the combination of colistin ( $2 \mu \mathrm{g} / \mathrm{mL})$ plus capsaicin (32 $\mu \mathrm{g} / \mathrm{mL})$.

(A): Differential gene volcano map. (B): KEGG enrichment analysis of up-regulation genes. (C): KEGG enrichment analysis of down-regulation genes; the color of the dot represents the size of the $Q$ value, and the size of the dot represents the number of differential genes. (D): Differentially expressed genes of ribosomes, multidrug efflux pump and outer membrane lipoprotein. Col: colistin alone; Col + Cap: the combination of colistin and capsaicin.

have been reported, such as destruction of bacterial cell membrane, loss of membrane potential, damage of ATP production and inhibition of the expression of virulence genes. When combined with antibiotics, some phytochemicals not only have direct antibacterial activity, but also show synergistic effect in vitro, thus changing the antibiotic resistance (Baskaran et al., 2016; Barbieri et al., 2017). Chelation of metal ions, inhibition of membrane-bound ATPase and changes of membrane 
permeability caused by plant compounds affect the normal physiological function of bacteria and lead to cell death (Subramani et al., 2017). Plant derived antimicrobial agents, such as resveratrol, coumarin, carvacrol, thymol, eugenol and catechins, play an antibacterial role by inhibiting the formation of biofilm and the ability to adhere to the host and destroying the cell membrane (Chen et al., 2016; Li et al., 2016). Essential oil is a secondary metabolite produced by aromatic plants and has significant antibacterial activity. It can interact with multiple targets, resulting in destruction of cell membrane, inhibition of protein synthesis and efflux pump activity (Mittal et al., 2019).

In our study, we found that capsaicin can significantly enhance the sensitivity of $A$. baumannii to colistin. On the one hand, capsaicin inhibits the biofilm formation of A. baumannii and destroys the outer membrane permeability, which may enable hydrophobic capsaicin molecules to pass through the lipopolysaccharide in the outer layer of bacteria and reach the target, thus playing a synergistic role. In addition, capsaicin promotes the expression of ribosomal synthesis genes, which is supposed to be the result of its inhibition of protein synthesis. At the same time, capsaicin can also inhibit the expression of drug efflux pump genes and the outer-membrane lipoprotein genes, which meant that the efflux, proliferation and adhesion ability of bacteria were weakened.

\section{CONCLUSION}

In conclusion, we report that the combination of capsaicin with colistin may be a viable alternative treatment option to combat colistin-resistant A. baumannii strains. This treatment reduces the dosage of colistin, thereby reducing the selective pressure on the target strains. One limitation of our study was that the clinical isolates collected did not include isolates that were naturally highly resistant to colistin. The discovery of capsaicin as a new colistin adjuvant highlights the huge potential of natural drug to treat bacterial infections. This may confirm the potential efficacy of capsaicin to treat clinical colistin-resistant A. baumannii.

\section{REFERENCES}

Armengol, E., Domenech, O., Fusté, E., Pérez-Guillén, I., Borrell, J. H., Sierra, J. M., et al. (2019). Efficacy of Combinations of Colistin with Other Antimicrobials Involves Membrane Fluidity and Efflux Machinery. Infect. Drug Resist. 12, 2031-2038. doi:10.2147/IDR.S207844

Barbieri, R., Coppo, E., Marchese, A., Daglia, M., Sobarzo-Sánchez, E., Nabavi, S. F., et al. (2017). Phytochemicals for Human Disease: An Update on Plant-Derived Compounds Antibacterial Activity. Microbiol. Res. 196, 44-68. doi:10.1016/ j.micres.2016.12.003

Basith, S., Cui, M., Hong, S., and Choi, S. (2016). Harnessing the Therapeutic Potential of Capsaicin and its Analogues in Pain and Other Diseases. Molecules 21. doi:10.3390/molecules21080966

Baskaran, S. A., Kollanoor-Johny, A., Nair, M. S., and Venkitanarayanan, K. (2016). Efficacy of Plant-Derived Antimicrobials in Controlling Enterohemorrhagic Escherichia coli Virulence In Vitro. J. Food Prot. 79, 1965-1970. doi:10.4315/ 0362-028X.JFP-16-104

\section{DATA AVAILABILITY STATEMENT}

The data presented in the study are deposited in the NCBI Sequence Read Archive (SRA), accession number SRR15633557 and SRR15633558.

\section{ETHICS STATEMENT}

The animal study was reviewed and approved by he Guidelines for the Care and Use of Laboratory Animals and procedures were approved by the Ethical Committee of Yangzhou University and the Laboratory Animal Management Committee of Yangzhou Province.

\section{AUTHOR CONTRIBUTIONS}

Author Contribtions: TG and GL contributed to the design of the study. TG, ML, YW, LY, and XS contributed to the acquisition of the data. TG, ML,HJ, and LY contributed to the analysis of the data. All authors contributed to data interpretation, drafting the manuscript, critically revising the manuscript for important intellectual content, and approved the final version of the manuscript.

\section{FUNDING}

This work was supported by the National Natural Science Foundation of China (82073611 and 82002186), the Natural Science Foundation of the Higher Education Institutions of Jiangsu Province (Grant number: 19KJB310002)

\section{ACKNOWLEDGMENTS}

We thank Kate Fox, DPhil, from Liwen Bianji, Edanz Group China (www.liwenbianji.cn/ac), for editing the English text of a draft of this manuscript.

Bassetti, M., Merelli, M., Temperoni, C., and Astilean, A. (2013). New Antibiotics for Bad Bugs: where Are We? Ann. Clin. Microbiol. Antimicrob. 12, 22. doi:10.1186/1476-0711-12-22

Brown, D. (2015). Antibiotic Resistance Breakers: Can Repurposed Drugs Fill the Antibiotic Discovery Void? Nat. Rev. Drug Discov. 14, 821-832. doi:10.1038/nrd4675

Ceri, H., Olson, M. E., Stremick, C., Read, R. R., Morck, D., and Buret, A. (1999). The Calgary Biofilm Device: New Technology for Rapid Determination of Antibiotic Susceptibilities of Bacterial Biofilms. J. Clin. Microbiol. 37, 1771-1776. doi:10.1128/JCM.37.6.1771-1776.1999

Chen, H., Li, H., Liu, Z., and Li, J. (2020). In Vitro and In Vivo Effects of the Polymyxin-Vorinostat Combination Therapy against Multidrug-Resistant Gram-Negative Pathogens. Microb. Drug Resist. 26, 1108-1119. doi:10.1089/ mdr.2019.0309

Chen, J., Yu, Y., Li, S., and Ding, W. (2016). Resveratrol and Coumarin: Novel Agricultural Antibacterial Agent against Ralstonia Solanacearum in Vitro and in Vivo, 21. Basel, Switzerland: Molecules. doi:10.3390/molecules21111501

Claeys, K. C., Fiorvento, A. D., and Rybak, M. J. (2014). A Review of Novel Combinations of Colistin and Lipopeptide or Glycopeptide Antibiotics for the 
Treatment of Multidrug-Resistant Acinetobacter Baumannii. Infect. Dis. Ther. 3, 69-81. doi:10.1007/s40121-014-0051-9

Dinc, G., Demiraslan, H., Elmali, F., Ahmed, S. S., Alp, E., and Doganay, M. (2015). Antimicrobial Efficacy of Doripenem and its Combinations with Sulbactam, Amikacin, Colistin, Tigecycline in Experimental Sepsis of CarbapenemResistant Acinetobacter Baumannii. New Microbiol. 38, 67-73.

Erfanimanesh, S., Eslami, G., Taherpour, A., and Hashemi, A. (2019). Capsaicin Inhibitory Effects on Vibrio cholerae Toxin Genes Expression. Avicenna J. Phytomed 9, 187-194.

Eze, E. C., Chenia, H. Y., and El Zowalaty, M. E. (2018). Acinetobacter Baumannii Biofilms: Effects of Physicochemical Factors, Virulence, Antibiotic Resistance Determinants, Gene Regulation, and Future Antimicrobial Treatments. Infect. Drug Resist. 11, 2277-2299. doi:10.2147/IDR.S169894

Fan, B., Guan, J., Wang, X., and Cong, Y. (2016). Activity of Colistin in Combination with Meropenem, Tigecycline, Fosfomycin, Fusidic Acid, Rifampin or Sulbactam against Extensively Drug-Resistant Acinetobacter Baumannii in a Murine Thigh-Infection Model. PloS one 11, e0157757. doi:10.1371/journal.pone.0157757

Han, M. L., Liu, X., Velkov, T., Lin, Y. W., Zhu, Y., Creek, D. J., et al. (2019). Comparative Metabolomics Reveals Key Pathways Associated with the Synergistic Killing of Colistin and Sulbactam Combination against Multidrug-Resistant Acinetobacter Baumannii. Front. Pharmacol. 10, 754. doi:10.3389/fphar.2019.00754

Harding, C. M., Hennon, S. W., and Feldman, M. F. (2018). Uncovering the Mechanisms of Acinetobacter Baumannii Virulence. Nat. Rev. Microbiol. 16, 91-102. doi:10.1038/nrmicro.2017.148

Jangra, M., Kaur, M., Tambat, R., Rana, R., Maurya, S. K., Khatri, N., et al. (2019). Tridecaptin M, a New Variant Discovered in Mud Bacterium, Shows Activity against Colistin- and Extremely Drug-Resistant Enterobacteriaceae. Antimicrob. Agents Chemother. 63. doi:10.1128/AAC.00338-19

Jangra, M., Randhawa, H. K., Kaur, M., Srivastava, A., Maurya, N., Patil, P. P., et al. (2018). Purification, Characterization and In Vitro Evaluation of Polymyxin A from Paenibacillus Dendritiformis: An Underexplored Member of the Polymyxin Family. Front. Microbiol. 9, 2864. doi:10.3389/ fmicb.2018.02864

Kaye, K. S., Pogue, J. M., Tran, T. B., Nation, R. L., and Li, J. (2016). Agents of Last Resort: Polymyxin Resistance. Infect. Dis. Clin. North. Am. 30, 391-414. doi:10.1016/j.idc.2016.02.005

Leelasupasri, S., Santimaleeworagun, W., and Jitwasinkul, T. (2018). Antimicrobial Susceptibility Among Colistin, Sulbactam, and Fosfomycin and a Synergism Study of Colistin in Combination with Sulbactam or Fosfomycin against Clinical Isolates of Carbapenem-Resistant Acinetobacter Baumannii. J. Pathog. 2018, 3893492. doi:10.1155/2018/3893492

Li, S., Yu, Y., Chen, J., Guo, B., Yang, L., and Ding, W. (2016). Evaluation of the Antibacterial Effects and Mechanism of Action of Protocatechualdehyde against Ralstonia Solanacearum, 21. Basel, Switzerland: Molecules. doi:10.3390/ molecules2 1060754

Lin, M. F., Lin, Y. Y., and Lan, C. Y. (2020). Characterization of Biofilm Production in Different Strains of Acinetobacter Baumannii and the Effects of Chemical Compounds on Biofilm Formation. PeerJ 8, e9020. doi:10.7717/peeri.9020

Magiorakos, A. P., Srinivasan, A., Carey, R. B., Carmeli, Y., Falagas, M. E., Giske, C. G., et al. (2012). Multidrug-resistant, Extensively Drug-Resistant and PandrugResistant Bacteria: an International Expert Proposal for Interim Standard Definitions for Acquired Resistance. Clin. Microbiol. Infect. 18, 268-281. doi:10.1111/j.1469-0691.2011.03570.x

Marini, E., Magi, G., Mingoia, M., Pugnaloni, A., and Facinelli, B. (2015). Antimicrobial and Anti-virulence Activity of Capsaicin against Erythromycin-Resistant, Cell-Invasive Group A Streptococci. Front. Microbiol. 6, 1281. doi:10.3389/fmicb.2015.01281

Matuschek, E., Åhman, J., Webster, C., and Kahlmeter, G. (2018). Antimicrobial Susceptibility Testing of Colistin - Evaluation of Seven Commercial MIC Products against Standard Broth Microdilution for Escherichia coli, Klebsiella pneumoniae, Pseudomonas aeruginosa, and Acinetobacter Spp. Clin. Microbiol. Infect. 24, 865-870. doi:10.1016/j.cmi.2017.11.020

Mi, H., Wang, D., Xue, Y., Zhang, Z., Niu, J., Hong, Y., et al. (2016). Dimethyl Sulfoxide Protects Escherichia coli from Rapid Antimicrobial-Mediated
Killing. Antimicrob. Agents Chemother. 60, 5054-5058. doi:10.1128/ AAC.03003-15

Mittal, R. P., Rana, A., and Jaitak, V. (2019). Essential Oils: An Impending Substitute of Synthetic Antimicrobial Agents to Overcome Antimicrobial Resistance. Curr. Drug Targets 20, 605-624. doi:10.2174/ 1389450119666181031122917

Ni, W., Shao, X., Di, X., Cui, J., Wang, R., and Liu, Y. (2015). In Vitro synergy of Polymyxins with Other Antibiotics for Acinetobacter Baumannii: a Systematic Review and Meta-Analysis. Int. J. Antimicrob. Agents 45, 8-18. doi:10.1016/ j.ijantimicag.2014.10.002

Pourhajibagher, M., Hashemi, F. B., Pourakbari, B., Aziemzadeh, M., and Bahador, A. (2016). Antimicrobial Resistance of Acinetobacter Baumannii to Imipenem in Iran: A Systematic Review and Meta-Analysis. Open Microbiol. J. 10, 32-42. doi:10.2174/1874285801610010032

Rolain, J. M., Kempf, M., Leangapichart, T., Chabou, S., Olaitan, A. O., Le Page, S., et al. (2016). Plasmid-Mediated Mcr-1 Gene in Colistin-Resistant Clinical Isolates of Klebsiella pneumoniae in France and Laos. Antimicrob. Agents Chemother. 60, 6994-6995. doi:10.1128/AAC.00960-16

Shin, J., Prabhakaran, V. S., and Kim, K. S. (2018). The Multi-Faceted Potential of Plant-Derived Metabolites as Antimicrobial Agents against MultidrugResistant Pathogens. Microb. Pathog. 116, 209-214. doi:10.1016/ j.micpath.2018.01.043

Srinivasan, K. (2016). Biological Activities of Red Pepper (Capsicum Annuum) and its Pungent Principle Capsaicin: A Review. Crit. Rev. Food Sci. Nutr. 56, 1488-1500. doi:10.1080/10408398.2013.772090

Subramani, R., Narayanasamy, M., and Feussner, K. D. (2017). Plant-derived Antimicrobials to Fight against Multi-Drug-Resistant Human Pathogens. 3 Biotech. 7, 172. doi:10.1007/s13205-017-0848-9

Thomas, V. M., Brown, R. M., Ashcraft, D. S., and Pankey, G. A. (2019). Synergistic Effect between Nisin and Polymyxin B against Pandrug-Resistant and Extensively Drug-Resistant Acinetobacter Baumannii. Int. J. Antimicrob. Agents 53, 663-668. doi:10.1016/j.ijantimicag.2019.03.009

Trebosc, V., Gartenmann, S., Tötzl, M., Lucchini, V., Schellhorn, B., Pieren, M., et al. (2019). Dissecting Colistin Resistance Mechanisms in Extensively DrugResistant Acinetobacter Baumannii Clinical Isolates. mBio 10. doi:10.1128/ mBio.01083-19

Upadhyay, A., Upadhyaya, I., Kollanoor-Johny, A., and Venkitanarayanan, K. (2014). Combating Pathogenic Microorganisms Using Plant-Derived Antimicrobials: a Minireview of the Mechanistic Basis. Biomed. Res. Int. 2014, 761741. doi:10.1155/2014/761741

Wareth, G., Neubauer, H., and Sprague, L. D. (2019). Acinetobacter Baumannii - a Neglected Pathogen in Veterinary and Environmental Health in Germany. Vet. Res. Commun. 43, 1-6. doi:10.1007/s11259-018-9742-0

Wei, W., Yang, H., Hu, L., Ye, Y., and Li, J. (2017). Activity of Levofloxacin in Combination with Colistin against Acinetobacter Baumannii: In Vitro and in a Galleria Mellonella Model. J. Microbiol. Immunol. Infect. 50, 821-830. doi:10.1016/j.jmii.2015.10.010

Xiong, Y. Q., Li, L., Zhou, Y., and Kraus, C. N. (2019). Efficacy of ARV-1502, a Proline-Rich Antimicrobial Peptide, in a Murine Model of Bacteremia Caused by Multi-Drug Resistant (MDR) Acinetobacter Baumannii. Molecules 24. doi:10.3390/molecules24152820

Yang, Y.-S., Lee, Y., Tseng, K.-C., Huang, W.-C., Chuang, M.-F., Kuo, S.-C., et al. (2016). In Vivo and In Vitro Efficacy of Minocycline-Based Combination Therapy for Minocycline-Resistant Acinetobacter Baumannii. Antimicrob. Agents Chemother. 60, 4047-4054. doi:10.1128/aac.02994-15

Yoshida, M., Reyes, S. G., Tsuda, S., Horinouchi, T., Furusawa, C., and Cronin, L. (2017). Time-programmable Drug Dosing Allows the Manipulation, Suppression and Reversal of Antibiotic Drug Resistance In Vitro. Nat. Commun. 8, 15589. doi:10.1038/ncomms15589

Zhang, S. S., Ni, Y. H., Zhao, C. R., Qiao, Z., Yu, H. X., Wang, L. Y., et al. (2018). Capsaicin Enhances the Antitumor Activity of Sorafenib in Hepatocellular Carcinoma Cells and Mouse Xenograft Tumors through Increased ERK Signaling. Acta Pharmacol. Sin 39, 438-448. doi:10.1038/ aps.2017.156

Zhou, Y., Guan, X., Zhu, W., Liu, Z., Wang, X., Yu, H., et al. (2014).Capsaicin Inhibits Porphyromonas Gingivalis Growth, Biofilm Formation, Gingivomucosal Inflammatory Cytokine Secretion, and In Vitro 
Osteoclastogenesis. Eur. J. Clin. Microbiol. Infect. Dis., 33, 211-219. doi:10.1007/s10096-013-1947-0

Conflict of Interest: The authors declare that the research was conducted in the absence of any commercial or financial relationships that could be construed as a potential conflict of interest.

Publisher's Note: All claims expressed in this article are solely those of the authors and do not necessarily represent those of their affiliated organizations, or those of the publisher, the editors and the reviewers. Any product that may be evaluated in this article, or claim that may be made by its manufacturer, is not guaranteed or endorsed by the publisher.

Copyright $\odot 2021$ Guo, Li, Sun, Wang, Yang, Jiao and Li. This is an open-access article distributed under the terms of the Creative Commons Attribution License (CC $B Y)$. The use, distribution or reproduction in other forums is permitted, provided the original author(s) and the copyright owner(s) are credited and that the original publication in this journal is cited, in accordance with accepted academic practice. No use, distribution or reproduction is permitted which does not comply with these terms. 